\title{
Low Loss Transmission Circular Polarizer for KU Band Application
}

\author{
Farman Ali Mangi ${ }^{1}$, Nafessa Shahani ${ }^{2}$, Deedar Ali Jamro ${ }^{1}$
}

\begin{abstract}
:
Low loss transmission circular polarizer is proposed for $\mathrm{Ku}$ band applications. The designed structure consists of two closely cross metallic strips which are based on FSS for 15.25 $\mathrm{GHz}$ and $15.28 \mathrm{GHz}$ applications. The right hand circular polarization (RHCP) and left handed circular polarization (LHCP) are obtained at $15.25 \mathrm{GHz}$ and at $15.28 \mathrm{GHz}$. The transmission loss through polarizer is important issue for high frequency applications. Due to transmission loss, new techniques are required to reduce the transmission loss of transmitted wave and achieve pure circular polarization. Meanwhile, low loss transmission has been achieved by using dual layer of strips to obtain perfect circular polarization at certain mentioned resonant frequencies. Theoretically, it is found that the outgoing waves through polarizer are perfect circular polarization at the distinct frequency ranges.
\end{abstract}

Keywords: Polarizer; Circular Polarization; Frequency selective surface; Quarter wave plate; Metamaterial.

\section{Introduction}

In modern microwave communication, circular polarization has received great interest in recent years. Precise, manipulation and detection of circular polarization of electromagnetic waves is important in modern technology. Artificially generating circular polarization is significantly more challenging in microwave communication technology. For instance, circular polarization is widely used in microwave communication and satellite communication systems. In addition, the $\mathrm{CP}$ achieves reflection effect, atmospheric absorption and lower susceptibility and intrinsically lower cross polarization discrimination.
An electronically steerable $\mathrm{CP}$ antenna array is more efficient for the inter-aircraft communication in terms of mobile nodes and wireless communication. The common application of antenna arrays is complex due to reduced level of practically-achievable output for extended millimetre wave communication network [1]. The CP antenna array approach was introduced for the use of a linearly polarised antenna array and electromagnetically coupled polarising wave plate. Dielectric polarisers [2], meander-line [3-5] and grid-plate [6] polarisers have been proposed to convert linear polarised electromagnetic waves to circular. $\mathrm{CP}$ antenna array is the combination of individual antenna elements which realize the directivity requirement and gain for the long distance [7-

\footnotetext{
${ }^{1}$ Department of Physics, Shah Abdul Latif University Khairpur, Sindh, Pakistan

${ }^{2}$ Institute of Chemistry, Shah Abdul Latif University Khairpur, Sindh, Pakistan

Corresponding Email: farman.mangi@ salu.edu.pk 
8]. In previous research contribution, the transmission type circular polarizers were proposed, such as U-shape split ring resonators, metallic helices, parallel plates, twisted Q-shaped metasurface, split ring resonator [9-10]. The four patch antennas were selected to produce $90^{\circ}$ phase shift and [11] introduces an elliptical $\mathrm{CP}$ dielectric resonator antenna array. In $[12,13]$, the thin dual-band polarizer was designed for satellite applications and meta-device with different functionalities is integrated.

The several known CP antennas composed of different multi-pot feed networks and radiation elements which makes them inefficient for practical application. The end fire antenna array are introduced to date [14, 15] which are constructed of seamless integration with linearly polarized. Polarization state of electromagnetic waves affect our daily life in order to consumer products to high technology applications [16]. The characteristics of a non-isotropic transparent material based on the electromagnetic waves incident upon a birefringent material.

If $\mathrm{E}$ field converted in two orthogonal components of $E_{x}$ and $E_{y}$ having same amplitude, the phase shift of any one component will result in circular polarization and satisfy the phase shift $90^{\circ}$ is known as quarter wave plate. The intensity of the incident electromagnetic wave does not change after propagating through a wave plate (only the polarisation state is changed). Wave plates are mostly considered as linearly birefringent, which means that the index of refraction differs along the two principal axes, which affects the phase shift of the orthogonal components differently [17].

Quarter wave plate possesses intriguing property to convert linear-to-circularly polarized waves when it is twisted at $45^{\circ}$ to the impinging polarizing plane. The unique characteristics of quarter wave plate is to change EM waves from linear-to-circularly polarized states. Most often, the manipulation or polarization control can be obtained with quarter wave plate [18]-[24]. The phase difference between outcome two orthogonal transmitted waves is quarter of the wavelength $90^{\circ}$ when impinged wave is linearly polarized at 450 and quarter wave plate has ability to change the impinged wave to circular polarization [25].

Frequency selective surface (FSSs) are developed as special filter and polarization transformer for microwave and millimetre waves. They are considered for many applications such as telecommunication, dichroic reflectors, waveguides and wireless security. [26-28]. FSSs can also be considered as polarizer [29-31] which has overall good performance and ease fabrication. The structure based on 15 x15 arrays of dual split ring resonators to demonstrate the transmission phase [32].

\section{Structure of Single Layer Polarizer}

First of all we have proposed single strip polarizer that operate at $13.20 \mathrm{GHz}$ is designed and fabricated to produce perfect right hand circular polarization. This single strip acts as a quarter-wave plate that transforms a linearly polarized incident wave into a circularly polarized transmitted wave. A single strip oriented at $45^{\circ}$ with $\lambda / 4$ arm length along the $x-y$ plane in simple unit cell. Whereas, the lengths of the single strip in $x-y$ directions is $7.5 \mathrm{~mm}$ and width is $2 \mathrm{~mm}$. Perfect Electric Conductor (PEC) material is used to the strip. The periodicity positions of the unit cell in three axes are $\mathrm{X}=28, \mathrm{Y}=, 28$ and $Z=25.25$, respectively. 


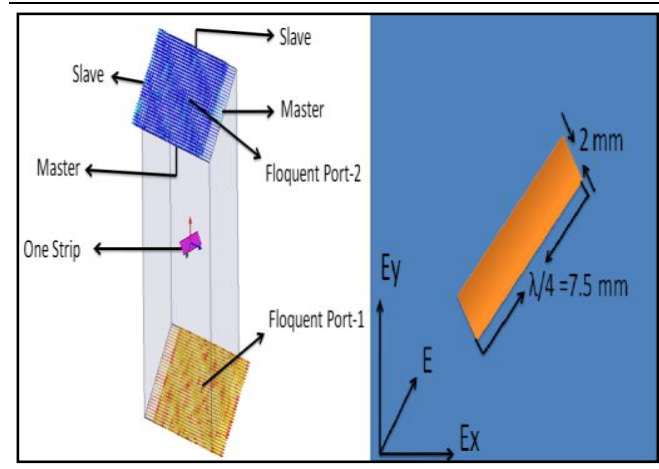

Fig. 1. View of circular polarizer based on single strip.

\section{Structure of Circular Polarizer}

Circular polarizer using FSS design for 15.25 GHz and $15.28 \mathrm{GHz}$ applications. Using this FSS to obtain CP at resonant frequencies. In this structure, two metallic strips are placed perpendicular at angle $+45^{\circ}$ and $-45^{\circ}$ along $x-y$ directions and separated at the distance of 7.2 mm perpendicularly from each other. The FSS polarizer is depicted in Fig.2. The proposed structure is constructed as cross dipole with $\lambda / 4$ arm length along $x-y$ axis. The length of each strips are selected $7.5 \mathrm{~mm}$ and $2 \mathrm{~mm}$ wide, respectively. The Perfect Electric Conductor (PEC) material is used to strips. The periodicity positions of the unit cell in three axes are $\mathrm{X}=33, \mathrm{Y}=, 33$ and $\mathrm{Z}=54$, respectively.

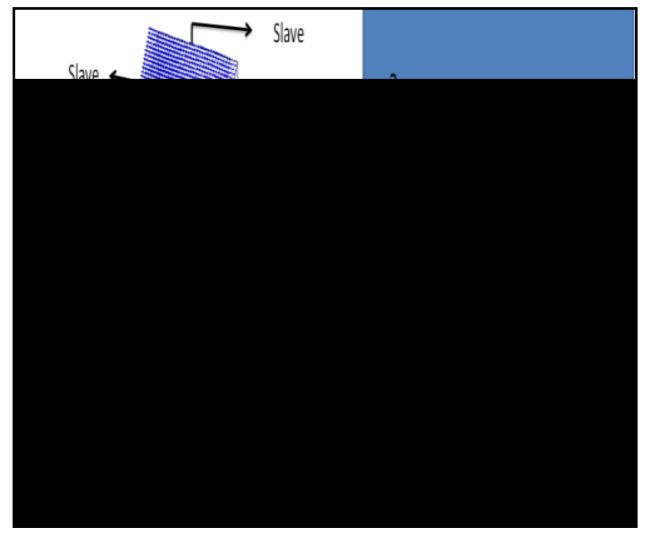

Fig. 2. View of Circular Polarizer based on two metallic strips.

\section{Simulation Analysis}

For simulation purpose, the HFSS software is used in terms of periodic boundary conditions. The impinged wave through floquet port one converted into circular polarized waves. The incident EM wave slanted at $45^{\circ}$ and emerge in to two decomposed orthogonal waves $\mathrm{E}_{\mathrm{x}}$ and $\mathrm{E}_{\mathrm{y}}$ to realize pure $\mathrm{CP}$ as shown in Fig.1. It can be observed that the transmission loss of designed structure $-3.2 \mathrm{~dB}$ at the $13.2 \mathrm{GHz}$ as depicted in Fig. 4. The axial ratio is 1.1 corresponds to phase difference of $90^{\circ}$ at resonant frequency of $13.2 \mathrm{GHz}$ mentioned in Fig.3 \& 5. The RHCP wave and LHCP wave are generated at distinct resonant frequencies. The transmission loss of dual Polarizer at the RHC polarization is $-1.8 \mathrm{~dB}$ and at LHC polarization is $-2.6 \mathrm{~dB}$ with respect to resonant frequencies 15.25 $\mathrm{GHz}$ and $15.28 \mathrm{GHz}$. Meanwhile, corresponding phase differences between outcome waves $\mathrm{E}_{\mathrm{x}}$ and $\mathrm{E}_{\mathrm{y}}$ are depicted in Fig. 5 and 8 . The simulated phase differences of outcome waves are satisfied at $90^{\circ}$ to achieve pure $\mathrm{CP}$ at resonant frequencies.

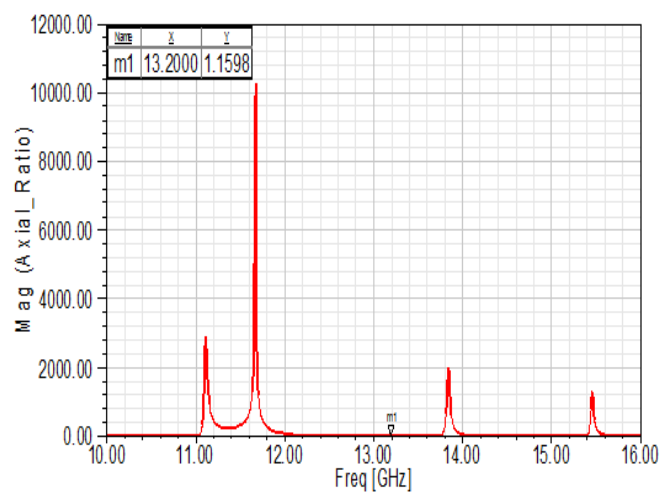

Fig. 3. Axial ratio at $13.20 \mathrm{GHz}$.

\section{Theoretical Results}

The HFSS software is used to analyze the obtained phase difference and magnitudes of output orthogonal components through designed low loss transmission circular polarizer. While, phase difference and transmission magnitudes of outcome waves 
can be directly calculated by formula $E_{\mathrm{x}} / \mathrm{E}_{\mathrm{y}}$ and $\Delta \phi=\mathrm{E}_{\mathrm{x}} \phi-\mathrm{E}_{\mathrm{y}} \phi$.

In Fig.1, the unit cell structure is depicted in Fig.1 which is oriented at $45^{\circ}$ in $\mathrm{x}-\mathrm{y}$ direction. The transmitted of two orthogonal waves is same at $13.20 \mathrm{GHz}$ which converts linear-to-circularly polarized wave. The calculated axial ratio of outcome two waves is 1.1 at $13.20 \mathrm{GHz}$ depicted in Fig. 3. The low loss transmission of the generated waves is noticed due to reflection of polarized constructed by single metallic strip. The generated orthogonal waves are same at 13.20 $\mathrm{GHz}$ but transmission loss about $-3.2 \mathrm{~dB}$ is observed. Obviously, the calculated transmission loss impact on the transmission power through single layer structure. The calculated phase difference of CP is $90^{\circ}$ at the 13.20 GHz which corresponds to axial ratio between two outcome waves as mentioned in Fig. 3.

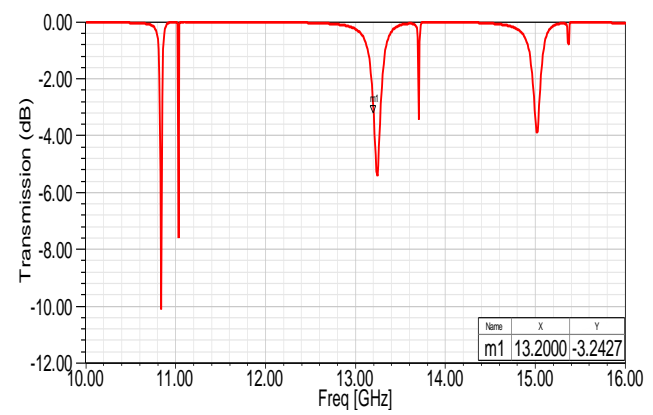

Fig. 4. Transmission magnitudes at $13.20 \mathrm{GHz}$.

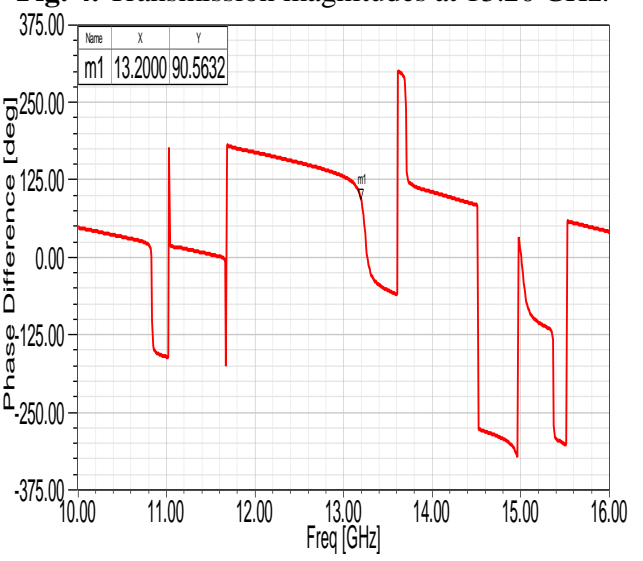

Fig. 5. Phase difference at $13.20 \mathrm{GHz}$.
The transmission loss of single-layer polarizer oriented at $45^{\circ}$ in $\mathrm{x}-\mathrm{y}$ direction is $3.2 \mathrm{~dB}$ as shown in Fig. 4. Furthermore, a innovative technique has been proposed to reduce transmission loss of single polarizer based on introducing the dual layer polarizer mounted perpendicularly to each other in the $\mathrm{x}-\mathrm{y}$ direction as shown in Fig. 2.

The magnitudes of output orthogonal components are similar at the frequency of 15.25 GHz and $15.28 \mathrm{GHz}$ to obtain the RHCP and LHCP. Nevertheless, it is necessary to obtain the circular polarization the phase difference of two orthogonal components $E_{x}$ and $E_{y}$ of $E$ field must be satisfied at $90^{\circ}$.The transmission axial ratio between two orthogonal components is 1.4 and 1.2 at 15.25 $\mathrm{GHz}$ and $15.28 \mathrm{GHz}$ as shown in Fig. 6. Whereas, the phase difference between two orthogonal components $\mathrm{E}_{\mathrm{x}}$ and $\mathrm{E}_{\mathrm{y}}$ is calculated by the phase difference formula of circular polarization.

The transmission loss after reflection from the Polarizer surface as shown in Fig.7 indicates the transmission magnitude of $\mathrm{E}$ components $\mathrm{E}_{\mathrm{x}}$ and $\mathrm{E}_{\mathrm{y}}$ in $\mathrm{dB}$ are -1.8 at 15.25 $\mathrm{GHz}$ and -2.60 at $15.28 \mathrm{GHz}$ as shown in Fig. 7. It means that the magnitude of both orthogonals $\mathrm{E}_{\mathrm{x}}$ and $\mathrm{E}_{\mathrm{y}}$ components are same. The transmission loss is observed -1.8 and $2.6 \mathrm{~dB}$ that effect the transmission of waves through polarizer. It is observed that the transmission loss of dual layer polarizer has been reduced against single polarizer as shown in Fig. 7. The circular polarization can be calculated by the following expression.

$$
\begin{aligned}
& \Delta \emptyset=\emptyset \mathbf{y}-\emptyset \mathbf{x} \\
& =\left\{\begin{array}{l}
+\left(\frac{\mathbf{1}}{\mathbf{2}}+\mathbf{2 n}\right) \boldsymbol{\pi}, \mathbf{n}=\mathbf{0}, \mathbf{1}, \mathbf{2}, \ldots \ldots \ldots \ldots \\
+\left(\left(\frac{\mathbf{1}}{\mathbf{2}}+\mathbf{2 n}\right) \boldsymbol{\pi}, \mathbf{n}=\mathbf{0}, \mathbf{1}, \mathbf{2}, \ldots \ldots \ldots \ldots\right.
\end{array}\right.
\end{aligned}
$$

It is observed circular polarization achieved when axial ratio value of two orthogonal components is satisfied 1.2 at certain mentioned frequencies and correspond to $89.9^{0}$ phase difference of two components. 
Therefore, we have achieved at about $99 \%$ $\mathrm{RCP}$ and $\mathrm{LCP}$ at the frequencies of $15.25 \mathrm{GHz}$ and $15.28 \mathrm{GHz}$ as shown in Fig.6 and 7. The phase difference is calculated by deducing the value form Fig. 8 and verified by above equation 1.

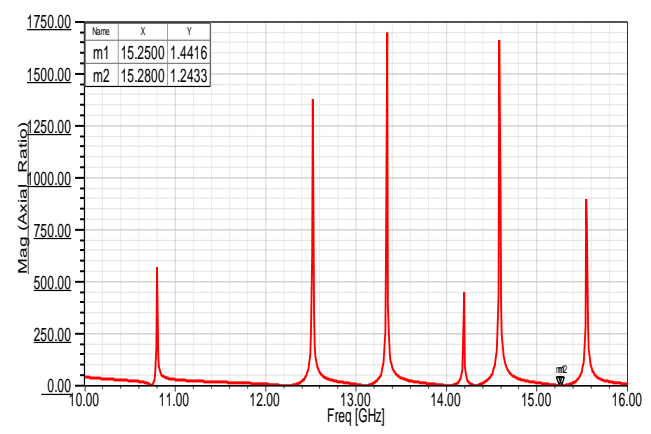

Fig. 6. Axial ratio is at $15.25 \mathrm{GHz}$ and $15.28 \mathrm{GHz}$.

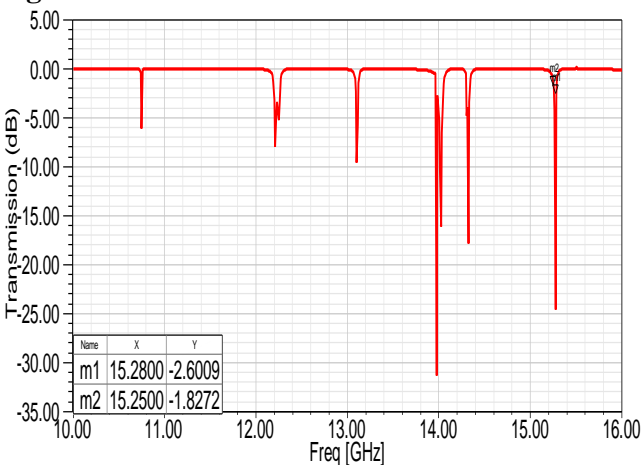

Fig. 7. Transmission magnitude at $15.25 \mathrm{GHz}$ and $15.28 \mathrm{GHz}$.

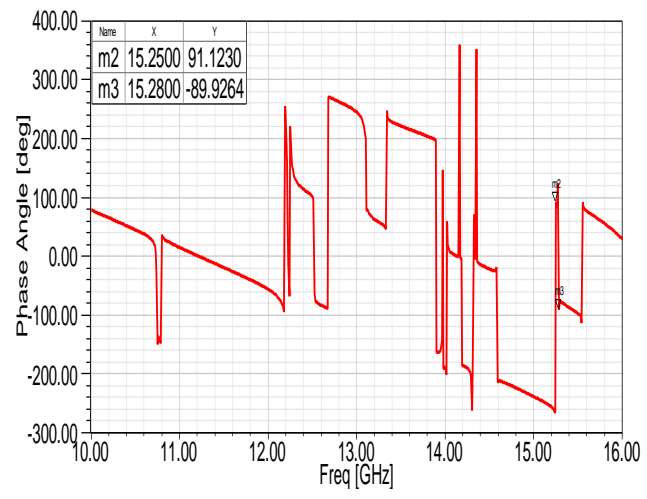

Fig. 8. Phase difference at $15.25 \mathrm{GHz}$ and $15.28 \mathrm{GHz}$.

\section{Conclusion}

In conclusion, we proposed the low loss transmission circular polarizer using two metallic strips to investigate the transmission characteristics with pure circular polarization at $15.25 \mathrm{GHz}$ and $15.28 \mathrm{GHz}$. The designed structure is based on FSS which generates the THCP wave and LHCP wave at resonance frequencies. The constructed model is simple and could be designed and fabricated easily by using HFSS software with high precision. The reflection and transmission characteristics are good features of the designed structure. In addition, the design techniques can be employed to construct the polarizers using double layer polarizers based on metallic strips or split ring resonators for millimetre, micrometre and terahertz frequencies. In future, this research can be carried out to compose polarizers using FSSs to extend the bandwidth and reduce the transmission loss.

\section{REFERENCES}

[1] V. Dyadyuk and Y. J. Guo, "Towards multi-gigabit ad-hoc wireless networks in the E-band", IEEE Global Symposium on Millimeter Waves, Sendai, Japan, 2009.

[2] L. Goldstone, "MM wave transmission polariser," In Antennas and Propagation Society International Symposium, 1979, vol. 17, pp. 606-609.

[3] L.Young, L. Robinson, and C. Hacking, "meander-line polarizer," IEEE Transactions on Antenna and propagation, vol. 21, pp. 376-378, 1973.

[4] R. S. Chu and K. M. Lee, "Analytical method of a multilayered meander-line polarizer plate with normal and oblique plane-wave incidence," IEEE Transactions on Antenna and Propagation, vol. 35, pp. 652-661, 1987.

[5] M. Mazur and W. Zieniutycz, "Multilayer meander line polarizer for $\mathrm{Ku}$ band," 13th International Conference on 
Microwaves, Radar and Wireless Communication, vol. 1, pp. 78-81, 2000.

[6] Z. Chen , X. Li, "Novel mixed grid-plate circular polarizers," 4th International Conference on Microwave and Millimeter Wave Technology (ICMMT 2004), Proc. pp. 66 - 69, Aug. 2004.

[7] W. Zhou, N. H. Noordin, N. Haridas, A. O. El-Rayis, A. T. Erdogan and T. Arslan, "A WiFi/4G compact feeding network for an 8-element circular antenna array," Loughborough Antennas and Propagation Conference (LAPC2011), pp. 1-4, 14-15 Nov. 2011.

[8] M. K. A. Rahim, T. Masri, O. Ayop and H. A. Majid, "Circular polarization array antenna," Asia-Pacific Microwave Conference, APMC 2008, pp. 1-4.

[9] M. Fartookzadeh and S. H. Mohseni Armaki, "Enhancement of Dual-Band Reflection-Mode Circular Polarizers Using Dual-Layer Rectangular Frequency Selective Surfaces," IEEE Transactions on Antennas and Propagation, vol. 64, no. 10, 2016.

[10] H. Cao, J. Liang, X. Wu and et al, "Dualband polarization conversion based on non-twisted Q-shaped metasurface," Optics Communications, vol. 370, 2016.

[11] S. L. S. Yang, R. Chair, A. A. Kishk, K.F. Lee and K.-M. Luk, "Study on sequential feeding networks for subarrays of circularly polarized elliptical dielectric resonator antenna," IEEE

Transactions on Antennas and Propagation, vol. 55, pp. 321-333, 2007.

[12] N. J. G. Fonseca and Cyril Mangenot, "High-Performance Electrically Thin Dual-Band Polarizing Reflective Surface for Broadband Satellite Applications," IEEE Transactions on Antennas and Propagation, vol. 64, no. 2, 2016.

[13]H. X. Xu, S. Sun, S. Tang and et al., "Dynamical control on helicity of electromagnetic waves by tunable metasurfaces," Sci. Rep, vol. 6, 2016.
[14] H. Uchimura, N. Shino, K. Miyazato, "Novel circular polarized antenna array substrates for 60GHz-band," IEEE MTT$S$ International Microwave Symposium Digest, 12-17 June 2005 Page(s):4.

[15] S. W. Wang et al., "A circular polarizer designed with a dielectric septum loading," IEEE Transactions on Microwave Theory and Techniques, vol. 52, no. 7, pp. 1719-1723, 2004.

[16] N. K. Grady et al., "Terahertz Meta Material for linear polarization conversion and anomalous refraction," Science vol. 340, no. 14 June 2013, pp. 1304,

[17] G. I. Kiani, and V. Dyadyuk, "Quarterwave plate polarizer based on frequency selective surface," European Microwave Conference, pp. 1361-1364, 28-30 Sept. 2010.

[18]D. Lerner, “A wave polarization converter for circular polarization," IEEE Trans. Antennas Propag., vol. AP13, no. 1, pp. 3-7, Jan. 1965.

[19] L. Young, L. Robinson, and C. Hacking, "Meander-line polarizer," IEEE Trans. Antennas Propag., vol. AP-21, no. 3, pp. 376-378, Mar. 1973.

[20] R.-S. Chu and K.-M. Lee, "Analytical method of a multilayered meander-line polarizer plate with normal and obliqueplane-wave incidence," IEEE Trans. Antennas Propag., vol. AP-35, no.6, pp. 652-661, Jun. 1987.

[21] A. K. Bhattacharyya and T. J. Chwalek, "Analysis of multilayered meander line polarizer," Int. J. Microw. MillimeterWave Comput.-Aided Eng., vol. 7, no. 6, pp. 442-454, 1997.

[22] M. Euler, V. Fusco, R. Cahill, and R. Dickie, "Comparison of frequencyselective screen- based linear to circular split-ring polarisation convertors," IET Microw., Antennas, Propag., vol. 4, no. 11, pp. 1764-1772, 2010. 
[23] M. Euler, V. Fusco, R. Cahill, and R. Dickie, "325 GHz single layer submillimeter wave fss based split slot ring linear to circular polarization convertor," IEEE Trans. Antennas Propag., vol. 58, no. 7, pp. 2457-2459, Jul. 2010.

[24]M. Joyal and J. Laurin, "Analysis and design of thin circular polarizers based on meander lines," IEEE Trans. Antennas Propag., vol. 60, no. 6, pp. 3007-3011, Jun. 2012

[25]C. Pfeiffer and A. Grbic, "MillimeterWave Transmitarrays for Wavefront and Polarization Control," IEEE Transactions on Microwave Theory and Techniques, vol. 61, no. 12, pp, 4407, december 2013,

[26]B. A. Munk, Frequency Selective Surfaces, Theory and Design, John Wiley \& Sons Inc, 2000.

[27] G. I. Kiani, A. R. Weily, and K. P. Esselle, "A novel absorb/transmit FSS for secure indoor wireless networks with reduced multipath fading," IEEE Microwave and Wireless Components Letters, 16, pp. 378-380, 2006.

[28] G. I. Kiani, K. L. Ford, K. P. Esselle, A. R. Weily, and C. J. Panagamuwa, "Oblique incidence performance of a novel frequency selective surface absorber," IEEE Transactions on Antennas and Propagation, vol. 55, pp. 2931-2934, 2007.

[29] A. Strikewerda et al., "Comparison of birefringent metamaterials and meaderline structure as quarter-wave plates at terahertz frequencies," Optics Express, 17, pp. 136-149, 2009.

[30]K. Karkkainen, and M. Stuchly, "Frequency selective surface as a polarisation transformer," IEE Proceedings on Microwaves, Antennas and Propagation, vol. 149, pp. 248-252, 2002.

[31] S. A. Winkler, W. Hong, M. Bozzi, and $\mathrm{K}$. $\mathrm{Wu}$, "A novel polarization rotating frequency selective surface based on substrate integrated waveguide technology," In Proceedings of the 39th European Microwave Conference, Rome, Italy, 2009.

[32] H. X. Xu, S. Tang, G. M. Wang and et al., "Multifunctional Microstrip Array Combining a Linear Polarizer and Focusing Metasurface," IEEE Transactions on Antennas and Propagation, vol. 64, No. 8, (2016). 